Article

\title{
Cyclizations Producing Hydrindanones with Two Methyl Groups at the Juncture Positions Mediated by Samarium Diiodide and Electrolysis
}

\author{
Masakazu Sono *, Natsuko Ise, Tsutomu Shoji and Motoo Tori * \\ Faculty of Pharmaceutical Sciences, Tokushima Bunri University, Yamashiro-cho, \\ Tokushima 770-8514, Japan \\ * Authors to whom correspondence should be addressed; E-Mails: sono@ph.bunri-u.ac.jp (M.S.); \\ tori@ph.bunri-u.ac.jp (M.T.); Tel.: +81-88-602-8462 (M.T.); Fax: +81-88-654-3051 (M.T.).
}

Received: 29 June 2012; in revised form: 8 August 2012 / Accepted: 3 September 2012 /

Published: 13 September 2012

\begin{abstract}
One-electron reductive intramolecular cyclization of enones with ketones or aldehydes mediated by samarium diiodide and electrolysis to afford cis-trimethylhydrindanolones. The reactions gave selectivities ranging from 1:1 to 100:0 depending on the conditions.
\end{abstract}

Keywords: samarium diiodide; electrolysis; cyclization; hydrindanone

\section{Introduction}

Samarium diiodide has been used for many synthetic reactions [1-4] and the mechanism of its reactions has also been studied [5,6]. We are interested in preparing bicyclic compounds such as perhydroindenes [7,8], perhydronaphthalenes [9], and guaianes [10] and have published some results in this area. Compounds bearing methyl groups at the juncture positions are interesting from the synthetic point of view. For example deoxopinguisone (1) [11,12] and $\alpha$-pinguisene (2) [13,14] (Figure 1) have four methyl groups in the bicyclo[4.3.0]nonane framework, all in a $\beta$-orientation; two of them are at the ring juncture positions, and have been synthetic targets $[15,16]$. We now planned to construct hydrindanone systems by 5 -exo mode cyclization, although such a case involving a cascade cyclization giving one substituent at the ring-juncture position was described by Procter and his group $[17,18]$. Electrolysis is easy to carry out using water as a solvent, which is environmentally 
benign $[19,20]$. We now describe the carbon-carbon bond formation reactions leading from $\mathrm{B}$ to $\mathrm{A}$ mediated by $\mathrm{SmI}_{2}$ as well as electrolysis for the synthesis of hydrindanones (Figure 1).

Figure 1. Target molecules and retrosynthesis.<smiles>CC1c2ccoc2C[C@]2(C)[C@H](C)CC[C@@]12C</smiles>

deoxopinguisone (1)<smiles>C=CC1=CC[C@]2(C)[C@H](C)CC[C@]2(C)[C@H]1C</smiles>

$\alpha$-pinguisene (2)

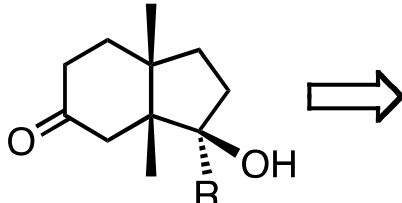

$\mathrm{R}=\mathrm{H}, \mathrm{Me}$

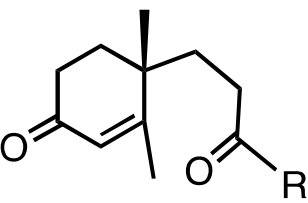

B

\section{Results and Discussion}

For the synthetic work we chose compounds 9 and $\mathbf{1 0}$ (Scheme 1), which were prepared starting from ketone 3. Several routine reactions afforded aldehyde $\mathbf{9}$ and ketone 10. This route can also be used in chiral form, because compound $\mathbf{3}$ is now commercially available and easy to prepare [21].

Scheme 1. Preparation of compounds 9 and $\mathbf{1 0 .}$<smiles>COC(=O)CC[C@]1(C)CCCCC1=O</smiles>

3
1) $\mathrm{PhMe}_{3} \mathrm{NBr}_{3}, \mathrm{THF}$ $0^{\circ} \mathrm{C}, 10 \mathrm{~min}$

2) $\mathrm{LiBr}, \mathrm{Li}_{2} \mathrm{CO}_{3}$ DMF<smiles>CC(=O)CC[C@]1(C)CCC=CC1=O</smiles>

4

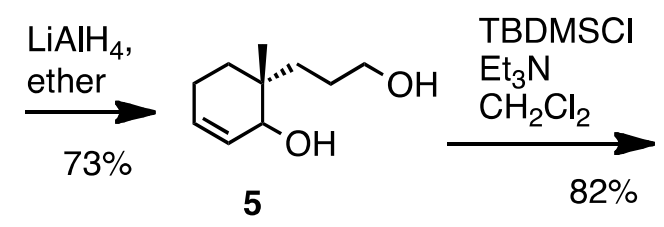

1) MeLi

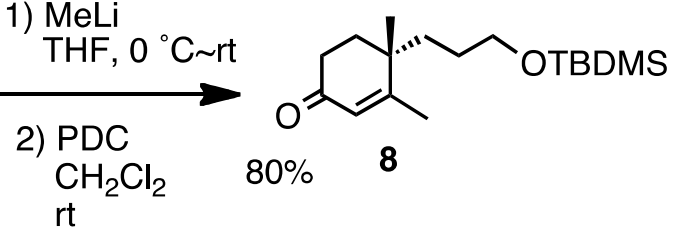<smiles>C[C@@]1(CCCO[As](C)(C)C)CCC=CC1O</smiles>

6

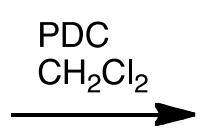

$87 \%$<smiles>CCCCOCCC[C@]1(C)CCC=CC1=O</smiles>

7

1) $\mathrm{AcOH}: \mathrm{H}_{2} \mathrm{O}: \mathrm{THF}$
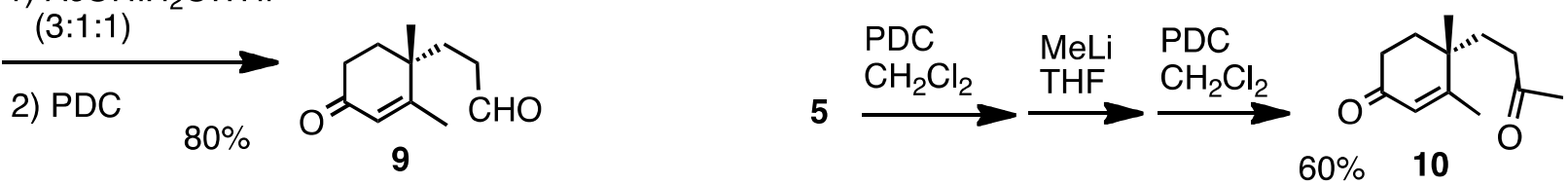

Aldehyde 9 was subjected to reaction with $\mathrm{SmI}_{2}$ (3 equiv.) in THF with or without additives. The results are shown in Table 1. The products were isomeric keto-alcohols $\mathbf{1 1}$ and 12, whose stereochemistries were established from the NOESY spectra. Without an additive, the reactions proceeded smoothly and both compounds were obtained in a ratio of 87:13 in favor of compound 11 at $0{ }^{\circ} \mathrm{C}$ (entry 1). When the reaction temperature was raised to rt (entry 2), the ratio of compound 12 increased to $31 \%$. The yields were always good. When $\mathrm{MeOH}$ was added as a proton source, the ratio was about 7:3 in favor of compound 11 (entries 3 and 4). The yield varied from $47 \%$ to $58 \%$. The reason why the yields were not so high is presumably due to the simple reduction of the double bond to give the corresponding dihydro derivatives, which were not isolated but detected in GC-MS. The ratio of 11 and 12 did not change very much when using HMPA (entries 7 and 8), however, when $\mathrm{NiI}_{2}$ was added (entries 9 and 10) the ratio of 12 was slightly increased [22]. 
Table 1. The reaction of aldehyde 9 with $\mathrm{SmI}_{2}$.

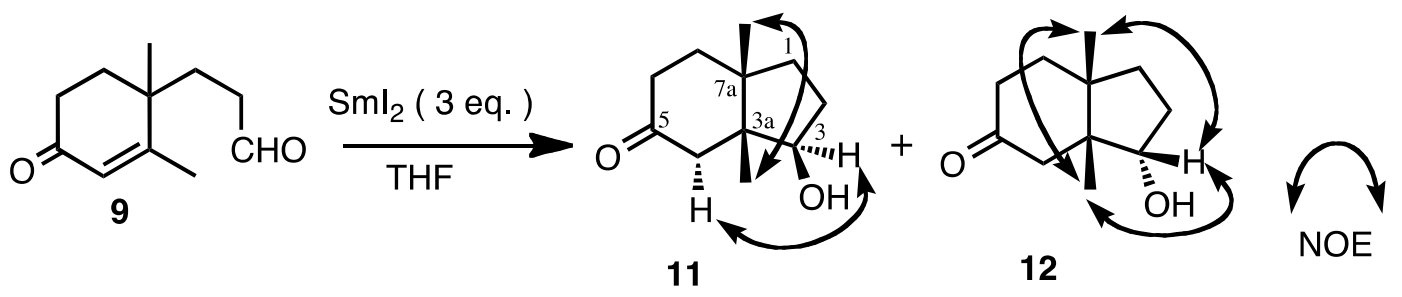

\begin{tabular}{cccccc}
\hline \multirow{2}{*}{ Entry } & \multirow{2}{*}{ Additives } & \multirow{2}{*}{ Temp $\left({ }^{\circ} \mathbf{C}\right)$} & Yield (\%) & \multicolumn{3}{c}{ Ratio } \\
\cline { 5 - 6 } 1 & none & 0 & quant. & 87 & 13 \\
2 & none & $\mathrm{rt}$ & quant. & 69 & 31 \\
3 & MeOH 2 equiv. & 0 & 58 & 72 & 28 \\
4 & MeOH 2 equiv. & $\mathrm{rt}$ & 47 & 69 & 31 \\
5 & HMPA 12 equiv. & 0 & 50 & 82 & 18 \\
6 & HMPA 12 equiv. & $\mathrm{rt}$ & 44 & 84 & 16 \\
7 & $\mathrm{NiI}_{2}$ & 0 & quant. & 75 & 25 \\
8 & $\mathrm{NiI}_{2}$ & $\mathrm{rt}$ & quant. & 56 & 44 \\
\hline
\end{tabular}

We next studied the reaction of ketone $\mathbf{1 0}$ under various conditions (Table 2). The products were isomeric keto-alcohols 13 and 14, and a mixture of bicyclic compounds 15, whose structures were determined by spectroscopic analyses. When ketone 10 was subjected to reaction with $\mathrm{SmI}_{2}$ without additive at $0{ }^{\circ} \mathrm{C}$ (entry 1), the products were 13 and 14 in a ratio of 84:16. In this case the major product had the hydroxy group in a $\beta$-orientation as determined by the NOESY spectrum (Table 2).

Table 2. The reaction of compound $\mathbf{1 0}$ with $\mathrm{SmI}_{2}$.

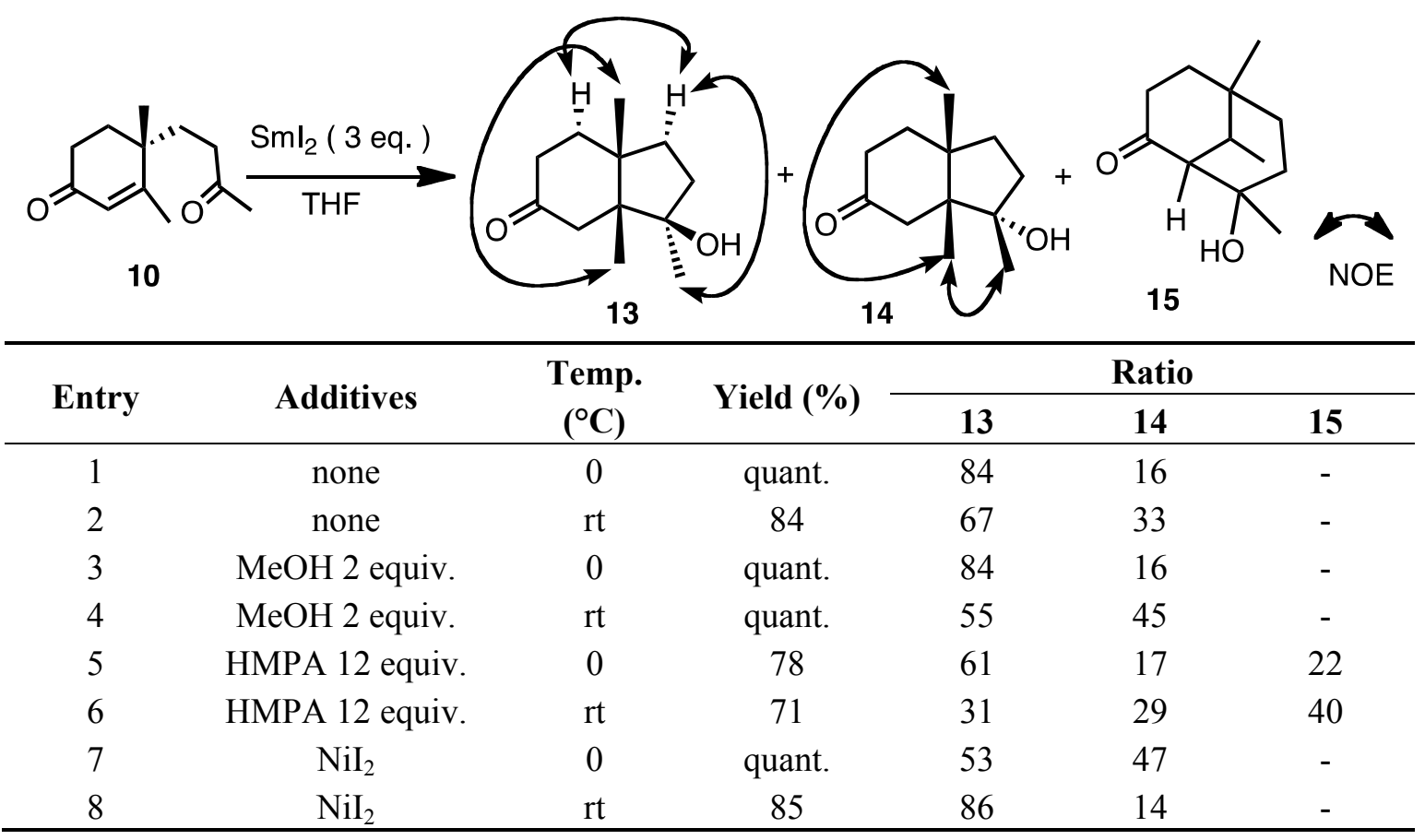

When the temperature was raised to rt (entry 2), the ratio of compound $\mathbf{1 4}$ increased. This tendency was the same as that of the aldehyde mentioned above. However, when HMPA was added, a third 
product 15 was also obtained (entries 5 and 6). The ratio of $\mathbf{1 5}$ was $40 \%$ of the products at $\mathrm{rt}$ from a total yield of $71 \%$ (entry 6 ). This product was formed by an aldol type condensation of the samarium enolate of the $\alpha, \beta$-unsaturated enone. When $\mathrm{NiI}_{2}$ was added, the yield was high and the ratio of compound 12 was slightly increased (entries 7 and 8) [22].

Electrolysis does not use organic solvents and expensive reagents, but rather water and electric power. In order to compare the selectivity, compounds $\mathbf{9}$ and $\mathbf{1 0}$ were subjected to electrolysis conditions as shown in Table 3. The yields were moderate and the ratio was 11:12 $=62: 38$ in the case of aldehyde 9. The results were not very different from those of samarium iodide reduction. However, in the case of ketone 10, only $\beta$-alcohol 13 was formed selectively (entry 2).

Table 3. The electrolysis of 9 and $\mathbf{1 0 .}$

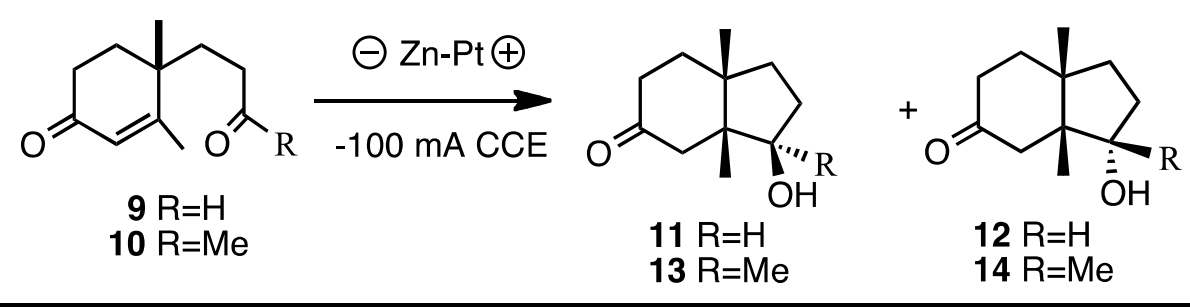

\begin{tabular}{ccccccc}
\hline Entry & Solvent & Additive & Time (h) & SM & Yield (\%) & Ratio \\
\hline 1 & ${ }^{t} \mathrm{BuOH}-\mathrm{H}_{2} \mathrm{O}(4: 6)$ & $\mathrm{Et}_{4} \mathrm{NTsO}$ & 2 & $\mathbf{9}$ & 53 & $\mathbf{1 1 : 1 2}=62: 38$ \\
2 & ${ }^{t} \mathrm{BuOH}-\mathrm{H}_{2} \mathrm{O}(4: 6)$ & $\mathrm{Et}_{4} \mathrm{NTsO}$ & 2 & $\mathbf{1 0}$ & 76 & $\mathbf{1 3 : 1 4}=100: 0$ \\
\hline
\end{tabular}

Comparison of the half-wave potentials of $\alpha, \beta$-unsaturated carbonyls with those of corresponding saturated carbonyl compounds has been extensively studied in electrochemistry [23]. The first waves of carbonyl groups, referred to as SCE., are $-2.45 \mathrm{~V}$ (cyclohexanone), $-2.25 \mathrm{~V}$ (methyl ethyl ketone), $-1.8 \mathrm{~V}$ (propionaldehyde), $-1.55 \mathrm{~V}$ (cyclohex-2-en-1-one), $-1.50 \mathrm{~V}$ (acrolein), and $-1.42 \mathrm{~V}$ (methyl vinyl ketone), respectively [23]. Therefore, the reduction of $\alpha, \beta$-unsaturated carbonyl moiety seems easier than that of the isolated ketone carbonyl group with electrochemistry, but the selectivity of the one-electron reduction of the carbonyl moiety using $\mathrm{SmI}_{2}$ depends on the stereoelectronic properties of the substrate [6]. Samarium transfers one-electron to the carbonyl group of the enone moiety to form a radical anion (C to D) (Figure 2). Then one more samarium atom reduces the ketone carbonyl group and the carbon-carbon bond is formed as shown by arrow $a$ to afford E (alkene first mechanism [2]). The hydroxy group is outside the bicyclic ring formed, because the samarium ion radical is large and the outside position is more energetically favored than inside of the ring. Thus, this configuration is more or less predominant. However, with HMPA as the additive, the reducing power must be higher than that with samarium alone [24], and the enone moiety is susceptible to reduction to afford a samarium enolate D. Then the aldol-type cyclization occurs to afford bicyclic anion radical F from D (shown by arrow $b$ in Figure 2). Further reduction of this anion radical $\mathrm{F}$ and protonation afford product 15. Electrolysis also creates a similar transition state leading to similar results. The reason why compound 10 produces 13 much more selectively is not clear at this stage. However, it is assumed that solvent molecules surround the methyl ketone carbonyl group resulting in the bulkier CO (solvent) moiety with consequent protrusion outside of the ring leading to the $\beta$-alcohol 13 [24]. 
Figure 2. Mechanisms of reductive cyclization $(\mathrm{R}=\mathrm{H}, \mathrm{Me})$.

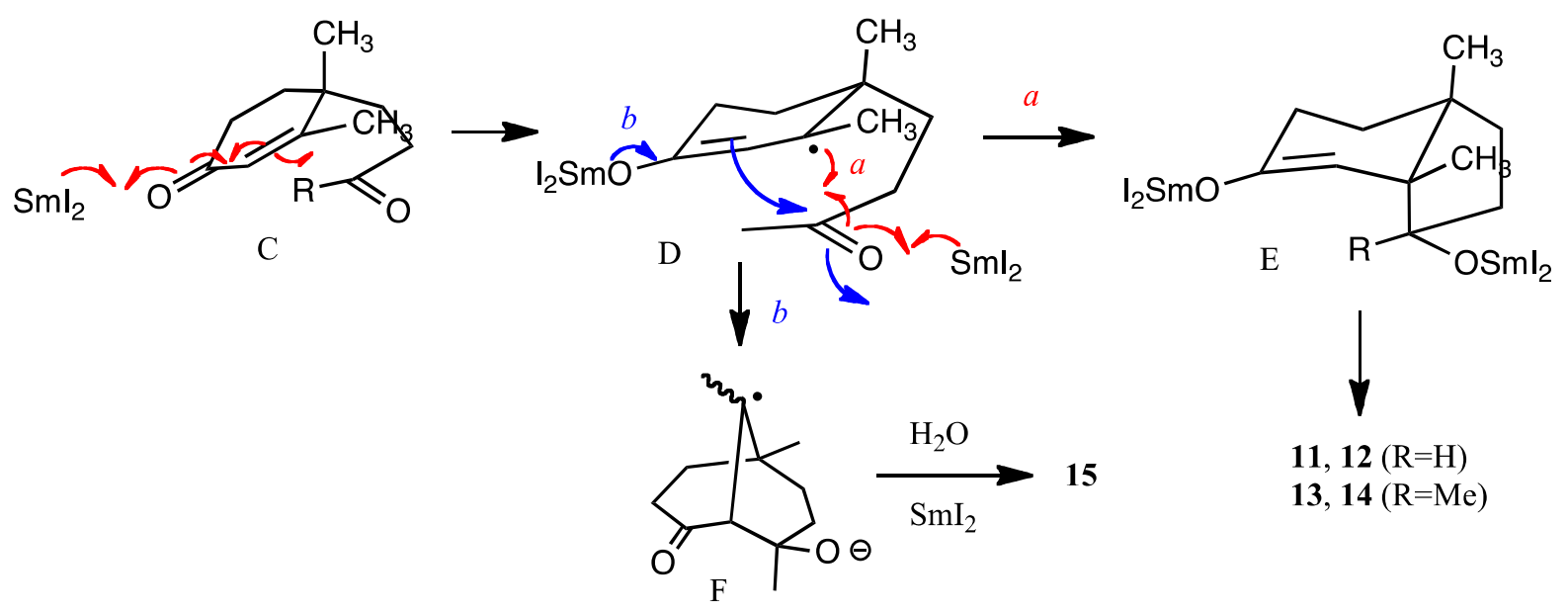

\section{Experimental}

\subsection{General}

IR spectra were measured on a JASCO FT/IR-5300 spectrophotometer. The ${ }^{1} \mathrm{H}$ and ${ }^{13} \mathrm{C}-\mathrm{NMR}$ spectra were taken using a Varian Unity 600 (at $600 \mathrm{MHz}$ and $150 \mathrm{MHz}$, respectively) and a Varian Unity 200 (200 MHz and $50 \mathrm{MHz}$, respectively) spectrometer. Mass spectra including high-resolution mass spectra were recorded on a JEOL JMS-700 MStation. A Chemcopak Nucleosil 50-5 column $(4.8 \times 250 \mathrm{~mm})$ was used for HPLC (JASCO pump system). For GC-MS an Agilent GC 6890 system equipped with a MS detector 5973 was used and the product ratios were determined by the area $\%$. Silica gel 60 (70-230 mesh, Fuji Silysia) was used for column chromatography and silica gel $60 \mathrm{~F}_{254}$ plates (Merck) were used for TLC.

\subsubsection{General Procedure for $\mathrm{Smi}_{2}$ Reduction}

A solution of substrate in dry THF was introduced into a solution of $\mathrm{SmI}_{2}$ at a certain temperature. Saturated solution of sodium potassium tartrate was added and the solvent was evaporated. The mixture was extracted with ether and worked up as usual. The residue was purified by silica-gel column chromatography.

\subsubsection{General Procedure for Electrolysis}

$\mathrm{Zn}$ and $\mathrm{Pt}$ were used for the cathode and anode, respectively, with $\mathrm{Et}_{4} \mathrm{NOT}_{\mathrm{N}}$ in $t \mathrm{BuOH}-\mathrm{H}_{2} \mathrm{O}(2: 3)$ $(20 \mathrm{~mL}), \mathrm{CCE}$ at $100 \mathrm{~mA}$ (from -1.5 to $-2.0 \mathrm{~V}$ vs. SCE) at rt. Work-up: benzene was added and most of the water was removed under reduced pressure. The residue was extracted with EtOAc and the organic layer was washed with Sat. $\mathrm{NaCl}$ solution. The organic layer was dried $\left(\mathrm{MgSO}_{4}\right)$, and the filtrate was evaporated to give a residue, which was purified by silica-gel column chromatography or HPLC. 


\subsection{Preparation of Methyl 3-(1-Methyl-2-oxocyclohex-3-enyl)propanoate (4)}

Ketone (3, $1 \mathrm{~g}, 0.5 \mathrm{mmol})$ was treated with phenyltrimethylammonium tribromide (1.9 g, $0.88 \mathrm{mmol})$ in THF $(5 \mathrm{~mL})$ at $0{ }^{\circ} \mathrm{C}$ for $10 \mathrm{~min}$. After the usual work-up, the residue $(925 \mathrm{mg})$ was successively treated with $\mathrm{LiBr}(585 \mathrm{mg}, 5.6 \mathrm{mmol})$ and $\mathrm{Li}_{2} \mathrm{CO}_{3}(148 \mathrm{mg}, 3.3 \mathrm{mmol})$ in DMF (4 mL) at $150{ }^{\circ} \mathrm{C}$ for $18 \mathrm{~h}$. Usual work-up and purification afforded enone 4 (684 mg, 69\%). Oil; IR (FT): 1730, 1660, $1630 \mathrm{~cm}^{-1}$; ${ }^{1} \mathrm{H}-\mathrm{NMR}\left(200 \mathrm{MHz}, \mathrm{CDCl}_{3}\right): \delta 1.09(3 \mathrm{H}, \mathrm{s}), 1.73-1.99$ (4H, m), 2.03-2.47 (4H, m), $3.65(3 \mathrm{H}, \mathrm{s}), 5.91(1 \mathrm{H}, \mathrm{dt}, J=10.2,1.8 \mathrm{~Hz}), 6.89(1 \mathrm{H}, \mathrm{dt}, J=10.2,4.0 \mathrm{~Hz}) ;{ }^{13} \mathrm{C}-\mathrm{NMR}(50 \mathrm{MHz}$, $\left.\mathrm{CDCl}_{3}\right)$ : $\delta 21.4\left(\mathrm{CH}_{3}\right), 22.9\left(\mathrm{CH}_{2}\right), 28.9\left(\mathrm{CH}_{2}\right), 31.1\left(\mathrm{CH}_{2}\right), 33.4\left(\mathrm{CH}_{2}\right), 43.7(\mathrm{C}), 51.4\left(\mathrm{CH}_{3}\right), 128.2$ (CH), $148.6(\mathrm{CH}), 173.9(\mathrm{C}), 203.1(\mathrm{C})$; MS m/z $196(\mathrm{M})^{+}, 181,165,136,110,68$ (base), 55, HRMS Found $m / z$ 196.1078. Calcd for $\mathrm{C}_{11} \mathrm{H}_{16} \mathrm{O}_{3}$ 196.1100.

\subsection{Preparation of 6-(3-Hydroxypropyl)-6-methylcyclohex-2-en-1-ol (5)}

A solution of enone $4(172 \mathrm{mg}, 0.88 \mathrm{mmol})$ in ether $(20 \mathrm{~mL})$ was treated with $\mathrm{LiAlH}_{4}(101 \mathrm{mg}$, $2.64 \mathrm{mmol})$ at $0{ }^{\circ} \mathrm{C}$ for $2 \mathrm{~h}$. Usual work-up afforded the diol $5(109 \mathrm{mg}, 73 \%)$ after purification as a mixture of diatereomers. Oil; IR (FT): 3330, 3020, $1660 \mathrm{~cm}^{-1} ;{ }^{1} \mathrm{H}-\mathrm{NMR}\left(200 \mathrm{MHz}, \mathrm{CDCl}_{3}\right): \delta 0.87$ $(3 \mathrm{H}, \mathrm{s}), 0.88(3 \mathrm{H}, \mathrm{s}), 1.2-1.7(12 \mathrm{H}, \mathrm{m}), 1.92-2.08(2 \mathrm{H}, \mathrm{m}), 3.03(2 \mathrm{H}, \mathrm{br} \mathrm{s}), 3.5-4.0(6 \mathrm{H}, \mathrm{m}), 5.5-6.0$ $(4 \mathrm{H}, \mathrm{m}) ;{ }^{13} \mathrm{C}-\mathrm{NMR}\left(50 \mathrm{MHz}, \mathrm{CDCl}_{3}\right): \delta 18.3\left(\mathrm{CH}_{3}\right), 21.3\left(\mathrm{CH}_{3}\right), 22.7\left(\mathrm{CH}_{2}\right), 22.8\left(\mathrm{CH}_{2}\right), 26.2\left(\mathrm{CH}_{2}\right)$, 26.6 $\left(\mathrm{CH}_{2}\right), 29.6\left(\mathrm{CH}_{2}\right), 30.3\left(\mathrm{CH}_{2}\right), 32.1\left(\mathrm{CH}_{2}\right), 35.1\left(\mathrm{CH}_{2}\right), 35.6(\mathrm{C}), 35.8(\mathrm{C}), 63.3\left(\mathrm{CH}_{2}\right), 71.7(\mathrm{CH})$, $72.2(\mathrm{CH}), 128.1(\mathrm{CH}), 128.6(\mathrm{CH}), 129.7(\mathrm{CH}), 129.8(\mathrm{CH})$; MS (CI) $m / z 169[\mathrm{M}-2+\mathrm{H}]^{+}, 153,135$ (base), 109; HRMS (CI) Found $m / z$ 169.1223 [M-2+H] $]^{+}$. Calcd for $\mathrm{C}_{10} \mathrm{H}_{17} \mathrm{O}_{2}$ 169.1229.

\subsection{Preparation of 6-(3-t-Butyldimethylsilyloxypropyl)-6-methylcyclohex-2-en-1-ol (6)}

A solution of diol $(5,480 \mathrm{mg}, 2.6 \mathrm{mmol})$ in $\mathrm{CH}_{2} \mathrm{Cl}_{2}(6 \mathrm{~mL})$ was treated with $\mathrm{Et}_{3} \mathrm{~N}(0.5 \mathrm{~mL}$, $3.4 \mathrm{mmol})$ and TBDMSCl (473 mg, $3.1 \mathrm{mmol})$ at $\mathrm{rt}$ for $18 \mathrm{~h}$. Usual work-up and purification afforded silyl ether 6 (658 mg, 82\%). Oil; IR (FT): 3400, $1660 \mathrm{~cm}^{-1} ;{ }^{1} \mathrm{H}-\mathrm{NMR}\left(200 \mathrm{MHz}, \mathrm{CDCl}_{3}\right): \delta 0.04$ $(6 \mathrm{H}, \mathrm{s}), 0.88(9 \mathrm{H}, \mathrm{s}), 0.89(3 \mathrm{H}, \mathrm{s}), 1.16-1.75(6 \mathrm{H}, \mathrm{m}), 1.92-2.04(2 \mathrm{H}, \mathrm{m}), 3.58(1 \mathrm{H}, \mathrm{d}, J=6.6 \mathrm{~Hz})$, $3.60(2 \mathrm{H}, \mathrm{t}, J=7.0 \mathrm{~Hz}), 3.74(1 \mathrm{H}$, br s$), 5.62-5.74(1 \mathrm{H}, \mathrm{m}), 5.79(1 \mathrm{H}, \mathrm{dt}, J=9.2,2.6 \mathrm{~Hz}){ }^{13} \mathrm{C}-\mathrm{NMR}$ $\left(50 \mathrm{MHz}, \mathrm{CDCl}_{3}\right): \delta-5.3\left(\mathrm{CH}_{3}\right), 18.4(\mathrm{C}), 21.4\left(\mathrm{CH}_{3}\right), 22.9\left(\mathrm{CH}_{2}\right), 26.0\left(\mathrm{CH}_{3}\right), 26.5\left(\mathrm{CH}_{2}\right), 29.7\left(\mathrm{CH}_{2}\right)$, $31.5\left(\mathrm{CH}_{2}\right), 35.7(\mathrm{C}), 64.0\left(\mathrm{CH}_{2}\right), 72.0(\mathrm{CH}), 128.3(\mathrm{CH}), 130.0(\mathrm{CH})$; MS (CI) m/z $284(\mathrm{M})^{+}, 267$, 227, 135 (base), 93, 83, 75; HRMS (CI) Found $m / z 284.2155(\mathrm{M})^{+}$. Calcd for $\mathrm{C}_{16} \mathrm{H}_{32} \mathrm{O}_{2} \mathrm{Si} 284.2172$.

\subsection{Preparation of 6-(3-t-Butyldimethylsilyloxypropyl)-6-methylcyclohex-2-en-1-one (7)}

A solution of alcohol $6(68 \mathrm{mg}, 0.24 \mathrm{mmol})$ was oxidized with PDC (270 $\mathrm{mg}, 0.72 \mathrm{mmol})$ in $\mathrm{CH}_{2} \mathrm{Cl}_{2}$ in the presence of molecular sieves $3 \mathrm{~A}(201 \mathrm{mg})$ at $\mathrm{rt}$ for $18 \mathrm{~h}$. Usual work-up afforded enone 7 (59 mg, 87\%) after purification. Oil; IR (FT): $1680 \mathrm{~cm}^{-1} ;{ }^{1} \mathrm{H}-\mathrm{NMR}\left(200 \mathrm{MHz}, \mathrm{CDCl}_{3}\right): \delta 0.03$ $(6 \mathrm{H}, \mathrm{s}), 0.87(9 \mathrm{H}, \mathrm{s}), 1.07(3 \mathrm{H}, \mathrm{s}), 1.32-1.63(4 \mathrm{H}, \mathrm{m}), 1.69-1.97(2 \mathrm{H}, \mathrm{m}), 2.19-2.50(2 \mathrm{H}, \mathrm{m}), 3.56$ $(2 \mathrm{H}, \mathrm{t}, J=7.0 \mathrm{~Hz}), 5.88(1 \mathrm{H}, \mathrm{dt}, J=10.2,2.0 \mathrm{~Hz}), 6.83(1 \mathrm{H}, \mathrm{dt}, J=10.2,4.0 \mathrm{~Hz}) ;{ }^{13} \mathrm{C}-\mathrm{NMR}(50 \mathrm{MHz}$, $\left.\mathrm{CDCl}_{3}\right): \delta-5.4\left(\mathrm{CH}_{3} \mathrm{X} 2\right), 18.2(\mathrm{C}), 21.7\left(\mathrm{CH}_{3}\right), 23.1\left(\mathrm{CH}_{2}\right), 25.9\left(\mathrm{CH}_{3} \mathrm{X} 3\right), 27.4\left(\mathrm{CH}_{2}\right), 32.3\left(\mathrm{CH}_{2}\right)$, $33.5\left(\mathrm{CH}_{2}\right), 44.2(\mathrm{C}), 63.5\left(\mathrm{CH}_{2}\right), 128.6(\mathrm{CH}), 148.5(\mathrm{CH}), 204.4(\mathrm{C})$; MS (CI) $m / z 283[\mathrm{M}+\mathrm{H}]^{+}, 267$, 225, 151 (base); CI-HRMS Found $m / z$ 283.2097 [M+H] $]^{+}$. Calcd for $\mathrm{C}_{16} \mathrm{H}_{31} \mathrm{O}_{2} \mathrm{Si}$ 283.2093. 


\subsection{Preparation of 4-(3-t-Butyldimethylsilyloxypropyl)-3,4-dimethylcyclohex-2-en-1-one (8)}

$\operatorname{MeLi}(0.88 \mathrm{~mL}, 1 \mathrm{mmol})$ was added to a stirred solution of enone 7 (39 $\mathrm{mg}, 0.22 \mathrm{mmol})$ and the mixture was stirred at $0^{\circ} \mathrm{C}$ for $22 \mathrm{~h}$. Usual work-up afforded a residue $36 \mathrm{mg}$ ), which was successively treated with PDC (140 mg, $0.36 \mathrm{mmol})$ in $\mathrm{CH}_{2} \mathrm{Cl}_{2}(4 \mathrm{~mL})$ at $\mathrm{rt}$ for $4 \mathrm{~h}$. Usual work-up and purification afforded enone 8 (33 mg, 80\%). Oil; IR (FT): 1680, $1620 \mathrm{~cm}^{-1} ;{ }^{1} \mathrm{H}-\mathrm{NMR}\left(200 \mathrm{MHz}, \mathrm{CDCl}_{3}\right): \delta 0.04$ $(6 \mathrm{H}, \mathrm{s}), 0.89(9 \mathrm{H}, \mathrm{s}), 1.14(3 \mathrm{H}, \mathrm{s}), 1.18-1.76(6 \mathrm{H}, \mathrm{m}), 1.90(3 \mathrm{H}, \mathrm{d}, J=1.2 \mathrm{~Hz}), 2.39(1 \mathrm{H}, J=6.4 \mathrm{~Hz})$, $2.43(1 \mathrm{H}, \mathrm{dd}, J=6.4,2.1 \mathrm{~Hz}), 3.5-3.7(2 \mathrm{H}, \mathrm{m}), 5.80(1 \mathrm{H}, \mathrm{d}, J=1.2 \mathrm{~Hz}) ;{ }^{13} \mathrm{C}-\mathrm{NMR}\left(50 \mathrm{MHz}, \mathrm{CDCl}_{3}\right)$ : $\delta-5.3\left(\mathrm{CH}_{3} \mathrm{X} 2\right), 18.3(\mathrm{C}), 20.0\left(\mathrm{CH}_{3}\right), 24.2\left(\mathrm{CH}_{3}\right), 25.9\left(\mathrm{CH}_{3} \mathrm{X} 3\right), 27.7\left(\mathrm{CH}_{2}\right), 33.4\left(\mathrm{CH}_{2}\right), 34.1\left(\mathrm{CH}_{2}\right)$, $34.8\left(\mathrm{CH}_{2}\right), 38.2(\mathrm{C}), 63.2\left(\mathrm{CH}_{2}\right), 127.2(\mathrm{CH}), 168.9(\mathrm{C}), 199.3(\mathrm{C})$; MS (CI) $\mathrm{m} / z 297[\mathrm{M}+\mathrm{H}]^{+}, 281$, 239 (base), 57, CI-HRMS Found $m / z$ 297.2226. Calcd for $\mathrm{C}_{17} \mathrm{H}_{33} \mathrm{O}_{2} \mathrm{Si}$ 297.2250.

\subsection{Preparation of 3-(1,2-Dimethyl-4-oxocyclohex-2-enyl)propanal (9)}

Enone 8 (67 mg, $0.23 \mathrm{mmol})$ was treated with $\mathrm{AcOH}: \mathrm{H}_{2} \mathrm{O}: \mathrm{THF}(3: 1: 1)(6 \mathrm{~mL})$ at $\mathrm{rt}$ overnight. Usual work-up afforded a residue (25 mg), which was subjected to PDC (978 mg, $2.6 \mathrm{mmol}$ ) oxidation in $\mathrm{CH}_{2} \mathrm{Cl}_{2}(30 \mathrm{~mL}$ in the presence of molecular sieves $3 \mathrm{~A}(903 \mathrm{mg})$ at $\mathrm{rt}$ for $2 \mathrm{~h}$. Usual work-up afforded enone 9 (33 mg, 80\%) after purification. Oil; IR (FT): 1720, 1670, $1620 \mathrm{~cm}^{-1}$; ${ }^{1} \mathrm{H}-\mathrm{NMR}$ $\left(200 \mathrm{MHz} \mathrm{CDCl}_{3}\right): \delta 1.16(3 \mathrm{H}, \mathrm{s}), 1.65-1.97(4 \mathrm{H}, \mathrm{m}), 1.88(3 \mathrm{H}, \mathrm{d}, J=1.3 \mathrm{~Hz}), 2.19-2.60(2 \mathrm{H}, \mathrm{m})$, $2.40(2 \mathrm{H}, \mathrm{t}, J=5.8 \mathrm{~Hz}), 5.82(1 \mathrm{H}, \mathrm{d}, J=1.3 \mathrm{~Hz}), 9.80(1 \mathrm{H}, \mathrm{t}, J=1.3 \mathrm{~Hz}) ;{ }^{13} \mathrm{C}-\mathrm{NMR}(50 \mathrm{MHz}$, $\left.\mathrm{CDCl}_{3}\right)$ : $\delta 19.9\left(\mathrm{CH}_{3}\right), 23.8\left(\mathrm{CH}_{3}\right), 29.8\left(\mathrm{CH}_{2}\right), 33.3\left(\mathrm{CH}_{2}\right), 33.9\left(\mathrm{CH}_{2}\right), 37.7(\mathrm{C}), 39.1\left(\mathrm{CH}_{2}\right), 127.9$ (CH), 167.3 (C), 198.9 (C), $201.3(\mathrm{CH})$; MS m/z $180\left(\mathrm{M}^{+}\right)$, 162, 152, 124, 109, 95 (base), 81, 67, 55; HRMS Found $m / z 180.1151(\mathrm{M})^{+}$. Calcd for $\mathrm{C}_{11} \mathrm{H}_{16} \mathrm{O}_{2} 180.1151$.

\subsection{Preparation of 4-(3-Oxobutyl)-3,4-dimethylcyclohex-2-en-1-one (10)}

Diol 5 (509 mg, 3 mmol) was oxidized with PDC (3.4 g, 9 mmol) in $\mathrm{CH}_{2} \mathrm{Cl}_{2}$ at $\mathrm{rt}$ overnight. Usual work-up afforded a residue $(97 \mathrm{mg})$, which was treated with MeLi $(9.3 \mathrm{~mL}, 10 \mathrm{mmol})$ in THF (30 mL) at $\mathrm{rt}$ for $16 \mathrm{~h}$. The residue after usual work-up was further treated with PDC (2.6 g, $7 \mathrm{mmol})$ in $\mathrm{CH}_{2} \mathrm{Cl}_{2}$ at rt overnight. Usual work-up afforded enone 5 (349 mg, 60\%) after purification. Oil; IR (FT): 1710, 1670, $1610 \mathrm{~cm}^{-1}$; ${ }^{1} \mathrm{H}-\mathrm{NMR}\left(200 \mathrm{MHz}, \mathrm{CDCl}_{3}\right): \delta 1.16(3 \mathrm{H}, \mathrm{s}), 1.64-1.97(4 \mathrm{H}, \mathrm{m}), 1.89(3 \mathrm{H}, \mathrm{d}$, $J=1.1 \mathrm{~Hz}), 2.17(3 \mathrm{H}, \mathrm{s}), 2.23-2.58(4 \mathrm{H}, \mathrm{m}), 5.83(1 \mathrm{H}, \mathrm{d}, J=1.1 \mathrm{~Hz}) ;{ }^{13} \mathrm{C}-\mathrm{NMR}\left(50 \mathrm{MHz}, \mathrm{CDCl}_{3}\right): \delta$ $19.9\left(\mathrm{CH}_{3}\right), 24.0\left(\mathrm{CH}_{3}\right), 30.1\left(\mathrm{CH}_{3}\right), 31.6\left(\mathrm{CH}_{2}\right), 33.3\left(\mathrm{CH}_{2}\right), 34.0\left(\mathrm{CH}_{2}\right), 37.8(\mathrm{C}), 38.5\left(\mathrm{CH}_{2}\right), 127.7$ (CH), 167.7 (C), 198.9 (C), 207.9 (C); MS m/z $194\left(\mathrm{M}^{+}\right)$, 176, 124 (base), 109, 95, 79, 67, 55; HRMS Found $m / z$ 194.1295 (M) ${ }^{+}$. Calcd for $\mathrm{C}_{12} \mathrm{H}_{18} \mathrm{O}_{2}$ 194.1307.

\section{9. $\left(3 R^{*}, 3 \mathrm{a} R^{*}, 7 \mathrm{a} S^{*}\right)-3-H y d r o x y-3 a, 7$ a-dimethylhexahydro-1H-inden-5(6H)-one (11)}

Oil; IR (FT): $3440,1710 \mathrm{~cm}^{-1}$; ${ }^{1} \mathrm{H}-\mathrm{NMR}\left(600 \mathrm{MHz}, \mathrm{CDCl}_{3}\right): \delta 0.90\left(3 \mathrm{H}, \mathrm{s}, 3 \mathrm{a}-\mathrm{CH}_{3}\right), 1.16(3 \mathrm{H}, \mathrm{s}$, 7a-CH $\mathrm{CH}_{3}, 1.55-1.74(4 \mathrm{H}, \mathrm{m}, \mathrm{H}-1,1,2 \beta, 7 \beta), 1.84(1 \mathrm{H}, \mathrm{ddd}, J=15.5,11.8,4.9 \mathrm{~Hz}, \mathrm{H}-7 \alpha), 2.12(1 \mathrm{H}, \mathrm{dd}$, $J=14.4,1.9 \mathrm{~Hz}, \mathrm{H}-4 \beta), 2.13-2.19$ (1H, m, H-2 $\alpha), 2.25$ (1H, dtd, $J=14.4,4.9,1.9 \mathrm{~Hz}, \mathrm{H}-6 \alpha), 2.29$ $(1 \mathrm{H}, \mathrm{dd}, J=14.4,1.9 \mathrm{~Hz}, \mathrm{H}-4 \alpha), 2.39$ (1H, dddd, $J=14.4,11.8,6.0,1.1 \mathrm{~Hz}, \mathrm{H}-6 \beta), 3.96$ (1H, dd, $J=8.5,6.6 \mathrm{~Hz}, \mathrm{H}-3) ;{ }^{13} \mathrm{C}-\mathrm{NMR}\left(150 \mathrm{MHz}, \mathrm{CDCl}_{3}\right): \delta 16.9\left(3 \mathrm{a}-\mathrm{CH}_{3}\right), 22.3\left(7 \mathrm{a}-\mathrm{CH}_{3}\right), 29.4(\mathrm{C}-2), 34.8$ (C-1), 36.8 (C-7), 37.8 (C-6), 41.5 (C-7a), 47.5 (C-4), 50.7 (C-3a), 78.0 (C-3), 212.5 (C-5); MS m/z 
$182\left(\mathrm{M}^{+}\right), 164,139,124,111$ (base), 96, 84, 79, 69, 55; HRMS Found $m / z 182.1298(\mathrm{M})^{+}$. Calcd for $\mathrm{C}_{11} \mathrm{H}_{18} \mathrm{O}_{2} 182.1307$.

3.10. (3S*,3a $\left.R^{*}, 7 \mathrm{a} S^{*}\right)-3-H y d r o x y-3 a, 7 a-d i m e t h y l h e x a h y d r o-1 H$-inden-5(6H)-one (12)

Oil; IR (FT): 3320, $1710 \mathrm{~cm}^{-1}$; ${ }^{1} \mathrm{H}-\mathrm{NMR}\left(600 \mathrm{MHz}, \mathrm{CDCl}_{3}\right): \delta 0.89\left(3 \mathrm{H}, \mathrm{s}, 3 \mathrm{a}-\mathrm{CH}_{3}\right), 1.04(3 \mathrm{H}, \mathrm{s}$, 7a-CH $\left.\mathrm{CH}_{3}\right), 1.53(1 \mathrm{H}, \mathrm{ddd}, J=12.9,10.2,6.5 \mathrm{~Hz}, \mathrm{H}-1 \beta), 1.60-1.71(2 \mathrm{H}, \mathrm{m}, \mathrm{H}-2 \alpha, 7 \alpha), 1.91-1.95(1 \mathrm{H}$,

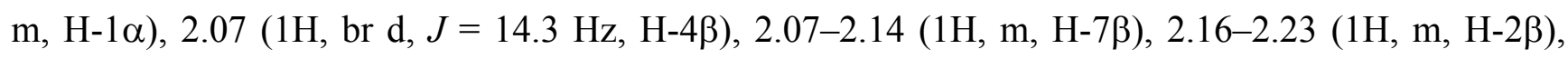

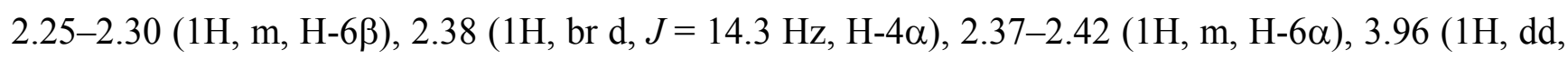
$J=7.7,4.5 \mathrm{~Hz}, \mathrm{H}-3) ;{ }^{13} \mathrm{C}-\mathrm{NMR}\left(150 \mathrm{MHz}, \mathrm{CDCl}_{3}\right): \delta 21.6\left(3 \mathrm{a}-\mathrm{CH}_{3}\right), 23.4\left(7 \mathrm{a}-\mathrm{CH}_{3}\right), 30.3(\mathrm{C}-2), 34.9$ (C-1), 35.8 (C-7), 37.6 (C-6), 41.6 (C-7a), 45.5 (C-4), 51.3 (C-3a), 81.9 (C-3), 213.0 (C-5); MS (EI) $m / z 182\left(\mathrm{M}^{+}\right), 164,139,124,111,95,84$ (base), 69, 55; HRMS Found $m / z 182.1308(\mathrm{M})^{+}$. Calcd for $\mathrm{C}_{11} \mathrm{H}_{18} \mathrm{O}_{2} 182.1307$.

\subsection{1. (3R*,3a $\left.R^{*}, 7 \mathrm{a} S^{*}\right)-3-H y d r o x y-3,3 a, 7 a-t r i m e t h y l h e x a h y d r o-1 H$-inden-5(6H)-one (13)}

Oil; IR (FT): 3480, $1710 \mathrm{~cm}^{-1} ;{ }^{1} \mathrm{H}-\mathrm{NMR}\left(600 \mathrm{MHz}, \mathrm{CDCl}_{3}\right): \delta 0.89\left(3 \mathrm{H}, \mathrm{s}, 3 \mathrm{a}-\mathrm{CH}_{3}\right), 1.15(3 \mathrm{H}, \mathrm{s}$, 7a-CH $\mathrm{CH}_{3}, 1.16\left(3 \mathrm{H}, \mathrm{s}, 3-\mathrm{CH}_{3}\right), 1.69$ (1H, ddd, $\left.J=14.2,7.4,4.9 \mathrm{~Hz}, \mathrm{H}-7 \beta\right), 1.77-1.81$ (3H, m, H-1,2,2), $1.84(1 \mathrm{H}, \mathrm{m}, \mathrm{H}-7 \alpha), 1.85-1.89$ (1H, m, H-1), 1.96 (1H, dd $J=14.0,1.1 \mathrm{~Hz}, \mathrm{H}-4 \beta), 2.20$ (1H, dddd, $J=17.3,9.3,4.9,1.1 \mathrm{~Hz}, \mathrm{H}-6 \beta), 2.28(1 \mathrm{H}, \mathrm{d}, J=14.0 \mathrm{~Hz}, \mathrm{H}-4 \alpha), 2.34(1 \mathrm{H}, \mathrm{ddd}, J=17.3,7.4,4.9 \mathrm{~Hz}$, $\mathrm{H}-6 \alpha) ;{ }^{13} \mathrm{C}-\mathrm{NMR}\left(150 \mathrm{MHz}, \mathrm{CDCl}_{3}\right): \delta 16.7\left(3 \mathrm{a}-\mathrm{CH}_{3}\right), 23.5\left(3-\mathrm{CH}_{3}\right), 26.6\left(7 \mathrm{a}-\mathrm{CH}_{3}\right), 36.1(\mathrm{C}-6), 36.3$ (C-7), 37.1 (C-2), 37.6 (C-1), 42.7 (C-7a), 49.7 (C-4), 50.9 (C-3a), 84.1 (C-3), 213.7 (C-5); MS m/z $196(\mathrm{M})^{+}, 178,150,139,123,111,84$ (base), 69, 55; HRMS Found $m / z 196.1452(\mathrm{M})^{+}$. Calcd for $\mathrm{C}_{12} \mathrm{H}_{20} \mathrm{O}_{2}$ 196.1464.

3.12. (3S*,3a $\left.R^{*}, 7 \mathrm{a} S^{*}\right)-3-H y d r o x y-3,3 a, 7 a-t r i m e t h y l h e x a h y d r o-1 H$-inden-5(6H)-one (14)

Oil; IR (FT): 3400, $1700 \mathrm{~cm}^{-1} ;{ }^{1} \mathrm{H}-\mathrm{NMR}\left(200 \mathrm{MHz}, \mathrm{CDCl}_{3}\right): \delta 0.86(3 \mathrm{H}, \mathrm{s}), 0.98(3 \mathrm{H}, \mathrm{s}), 1.18$ $(3 \mathrm{H}, \mathrm{s}), 1.40-1.50(3 \mathrm{H}, \mathrm{m}), 1.54-1.61(2 \mathrm{H}, \mathrm{m}), 1.71-1.79(2 \mathrm{H}, \mathrm{m}), 1.81-1.85(1 \mathrm{H}, \mathrm{m}), 1.88(1 \mathrm{H}, \mathrm{d}$, $J=11.8 \mathrm{~Hz}), 1.88-1.94(1 \mathrm{H}, \mathrm{m}) ;{ }^{13} \mathrm{C}-\mathrm{NMR}\left(50 \mathrm{MHz}, \mathrm{CDCl}_{3}\right): \delta 17.2\left(\mathrm{CH}_{3}\right), 22.9\left(\mathrm{CH}_{3}\right), 26.8\left(\mathrm{CH}_{3}\right)$, $35.1\left(\mathrm{CH}_{2}\right), 35.4\left(\mathrm{CH}_{2}\right), 35.7\left(\mathrm{CH}_{2}\right), 36.6\left(\mathrm{CH}_{2}\right), 42.6\left(\mathrm{CH}_{2}\right), 44.2(\mathrm{C}), 53.4(\mathrm{C}), 92.3(\mathrm{C}), 213.5(\mathrm{C})$; MS $m / z 196\left(\mathrm{M}^{+}\right), 178,151,139,125,109,95,84$ (base), 69, 55; HRMS Found $m / z 196.1460(\mathrm{M})^{+}$. Calcd for $\mathrm{C}_{12} \mathrm{H}_{20} \mathrm{O}_{2}$ 196.1463.

\subsection{8-Hydroxy-5,8,9-trimethylbicyclo[3.3.1]nonan-2-one (Mixture of Diatereoisomers) (15)}

Oil; IR (FT): 3420, $1700 \mathrm{~cm}^{-1}$; ${ }^{1} \mathrm{H}-\mathrm{NMR}\left(200 \mathrm{MHz}, \mathrm{CDCl}_{3}\right): \delta 0.90(0.25 \mathrm{H}, \mathrm{s}), 0.93(0.75 \mathrm{H}, \mathrm{d}$, $J=7.1 \mathrm{~Hz}), 0.96(0.75 \mathrm{H}, \mathrm{s}), 1.08(0.25 \mathrm{H}, \mathrm{s}), 1.40(0.75 \mathrm{H}, \mathrm{s}), 1.42(0.25 \mathrm{H}, \mathrm{s}) ;{ }^{13} \mathrm{C}-\mathrm{NMR}(150 \mathrm{MHz}$, $\left.\mathrm{CDCl}_{3}\right): \delta 28.0,28.7,31.4,35.8,38.2,39.5,40.5,65.5,66.6,69.9,71.8,214.1(\mathrm{CO})$; HRMS Found $\mathrm{m} / z \mathrm{z} 196.1465(\mathrm{M})^{+}$. Calcd for $\mathrm{C}_{12} \mathrm{H}_{20} \mathrm{O}_{2}$ 196.1463. 


\section{Conclusions}

We have developed methods to create cis-fused trimethylated hydrindanones in excellent- to high-yields by intra-molecular 5-exo cyclization mediated by $\mathrm{SmI}_{2}$ and electrolysis.

\section{Acknowledgments}

We thank Masami Tanaka and Yasuko Okamoto, Tokushima Bunri University, for measurement of $600 \mathrm{MHz}$ NMR and mass spectra, respectively.

\section{References}

1. Curran, D.P.; Porter, N.A.; Giese, B. Stereochemistry of Radical Reactions; Wiley-VCH Verlag GmbH: Weinheim, Germany, 1996.

2. Procter, D.J.; Flowers, R.A.; Skrydstrup, T. Organic Synthesis Using Samarium Diiodide: A Practical Guide; The Royal Society of Chemistry: Cambridge, UK, 2010.

3. Molander, G.A. Application of lanthanide reagents in organic synthesis. Chem. Rev. 1992, 92, $29-68$.

4. Molander, G.A.; Harris, C.R. Sequencing reactions with samarium (II) iodide. Chem. Rev. 1996, 96, 307-338.

5. Flowers, R.A.; Prasad, E. Samarium (II) based reductants. In Handbook on the Physics and Chemistry of Rare Earths; Gschneidner, K.A., Jr., Bunzli, J.C., Pecharsky, V.K., Eds.; Elsevier: Amsterdam, The Netherlands, 2006; Volume 36, pp. 393-473.

6. Sono, M.; Hanamura, S.; Furumaki, M.; Murai, H.; Tori, M. First direct evidence of radical intermediates in samarium diiodide induced cyclization by ESR spectra. Org. Lett. 2011, 13, 5720-5723.

7. Sono, M.; Nakashiba, Y.; Nakashima, K.; Tori, M. Cyclization into hydrindanones using samarium diiodide. J. Org. Chem. 2000, 65, 3099-3106.

8. Sono, M.; Hashimoto, A.; Nakashima, K.; Tori, M. Total synthesis of coronafacic acid through 6-endo-trig mode intramolecular cyclization of an enone-aldehyde to a hydrindanone using samarium (II) iodide. Tetrahedron Lett. 2000, 41, 5115-5118.

9. Sono, M.; Onishi, S.; Tori, M. Cyclization into perhydronaphthalenones using samarium diiodide. Tetrahedron 2003, 59, 3385-3395.

10. Sono, M.; Tatara, H.; Ise, N.; Takaoka, S.; Tori, M. Preparation of seven- and eight-membered rings by carbon-carbon bond formation induced by samarium diiodide: 7-endo- and 8-endo-trig type cyclization to bicyclo[5.3.0]decanolone, bicyclo[5.4.0]undecanolone, and bicyclo[6.4.0]dodecanolone. Tetrahedron 2008, 64, 11096-11104.

11. Benešová, V.; Samek, Z.; Herout, V.; Šorm, F. On terpenes. CXCVIII. Isolation and structure of pinguisone from Aneura pinguis. Collec. Czeck. Chem. Commun. 1969, 34, 582-592.

12. Nagashima, F.; Nishioka, E.; Kameo, K.; Nakagawa, C.; Asakawa, Y. Terpenoids and aromatic compounds from selected Ecuadorian liverworts. Phtochemistry 1991, 30, 215-217.

13. Asakawa, Y.; Toyota, M.; Takemoto, T. Sesquiterpenes from Porella species. Phytochemistry 1978, 17, 457-460. 
14. Fukuyama, Y.; Tori, M.; Wakamatsu, M.; Asakawa, Y. Norpinguisone methyl ester and norpinguisanolide, pinguisane-type norsesquiterpenoids from Porella elegantula. Phytochemistry 1988, 27, 3557-3561.

15. Tori, M.; Makino, C.; Hisazumi, K.; Sono, M.; Nakashima, K. Synthesis of a homochiral ketone having a pinguisane skeleton using phenylethylamine as a chiral auxiliary: A formal total synthesis of deoxopinguisone. Tetrahedron: Asymmetry 2001, 12, 301-307.

16. Shiina, J.; Oikawa, M.; Nakamura, K.; Obata, R.; Nishiyama, S. Synthesis of pinguisane-type sesquiterpenoids acutifolone A, pinguisenol, and bisacutifolones by a Diels-Alder dimerization reaction. Eur. J. Org. Chem. 2007, doi:10.1002/ejoc.200700522.

17. Findley, T.J.K.; Sucunza, D.; Miller, L.C.; Davies, D.T.; Procter, D.J. A flexible, stereoselective approach to the decorated cis-hydrindane skeleton: Synthesis of the proposed structure of faurinone. Chem. Eur. J. 2008, 14, 6862-6865.

18. Helm, M.D.; Sucunza, D.; Da Silva, M.; Helliwell, M.; Procter, D.J. SmI 2 -mediated dialdehyde cyclization cascades. Tetrahedron Lett. 2009, 50, 3224-3226.

19. Sono, M.; Shoji, T.; Tamaki, T.; Kishi, S.; Tori, M. The stereochemistry of electrolysis and samarium diiodide-induced cyclization between carbonyl and enone system in inter- and intramolecular coupling. Heterocycles 2007, 72, 517-528.

20. Shono, T. Electroorganic Synthesis; Academic Press: London, UK, 1991.

21. Pfau, M.; Revial, G.; Guingant, A.; d'Angelo, J. Enantioselective synthesis of quaternary carbon centers through Michael-type alkylation of chiral imines. J. Am. Chem. Soc. 1985, 107, 273-274.

22. Machrouhi, F.; Hamann, B.; Namy, J.L.; Kagan, H. Improved reactivity of diiodosamarium by catalysis with transition metal salts. Synlett 1996, 633-634.

23. Wagenknecht, J.H.; Eberson, L.; Utley, J.H.P. Carbonyl Compounds. In Organic Electrochemistry, 3rd ed.; Lund, H., Baizer, M.M., Eds.; Marcel Dekker Inc.: New York, NY, USA, 1991; pp. 433-464.

24. Prasad, E.; Flowers, R.A. Reduction of ketones and alkyl iodides by $\mathrm{SmI}_{2}$ and $\mathrm{Sm}(\mathrm{II})-\mathrm{HMPA}$ complexes. Rate and mechanistic studies. J. Am. Chem. Soc. 2002, 124, 6895-6899.

Sample Availability: Not available.

(C) 2012 by the authors; licensee MDPI, Basel, Switzerland. This article is an open access article distributed under the terms and conditions of the Creative Commons Attribution license (http://creativecommons.org/licenses/by/3.0/). 